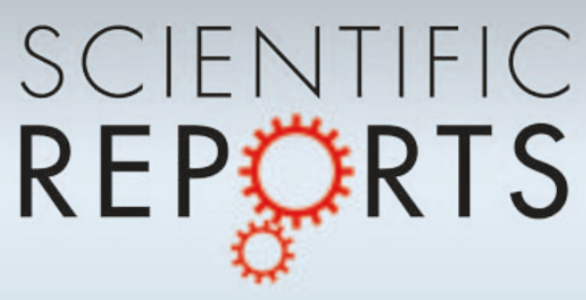

OPEN

SUBJECT AREAS:

ATOMIC AND

MOLECULAR PHYSICS

MAGNETIC MATERIALS AND

DEVICES

THEORETICAL PHYSICS

SUPERCONDUCTING MATERIALS

Received

5 October 2011

Accepted

16 April 2012

Published

1 May 2012

Correspondence and requests for materials should be addressed to

Y.L. (y4li@ucsd.edu)

\section{The J-triplet Cooper pairing with magnetic dipolar interactions}

\author{
Yi Li \& Congjun Wu \\ Department of Physics, University of California, San Diego, La Jolla, CA 92093.
}

Recently, cold atomic Fermi gases with the large magnetic dipolar interaction have been laser cooled down to quantum degeneracy. Different from electric-dipoles which are classic vectors, atomic magnetic dipoles are quantum-mechanical matrix operators proportional to the hyperfine-spin of atoms, thus provide rich opportunities to investigate exotic many-body physics. Furthermore, unlike anisotropic electric dipolar gases, unpolarized magnetic dipolar systems are isotropic under simultaneous spin-orbit rotation. These features give rise to a robust mechanism for a novel pairing symmetry: orbital $p$-wave $(L=1)$ spin triplet $(S$ $=1$ ) pairing with total angular momentum of the Cooper pair $J=1$. This pairing is markedly different from both the ${ }^{3} \mathrm{He}-\mathrm{B}$ phase in which $J=0$ and the ${ }^{3} \mathrm{He}-A$ phase in which $J$ is not conserved. It is also different from the $p$-wave pairing in the single-component electric dipolar systems in which the spin degree of freedom is frozen.

U ltracold atomic and molecular systems with electric and magnetic dipolar interactions have become the research focus in cold atom physics ${ }^{1-7}$. When dipole moments are aligned by external fields, dipolar interactions exhibit the $d_{r^{2}}-3 z^{2}$-type anisotropy. The anisotropic Bose-Einstein condensations of dipolar bosons (e.g. ${ }^{52} \mathrm{Cr}$ ) have been observed ${ }^{8-11}$. For the fermionic electric dipolar systems,$^{40} \mathrm{~K}-{ }^{87} \mathrm{Rb}$ has been cooled down to nearly quantum-degeneracy ${ }^{3}$. Effects of the anisotropic electric dipolar interaction on the fermion manybody physics have been extensively investigated. In the Fermi liquid theory, both the single particle properties and collective excitations exhibit the $d_{r^{2}}-3 z^{2}$ anisotropy ${ }^{12-17}$. In the single-component Fermi systems, the leading order Cooper pairing instability lies in the $p$-wave channel, which is the simplest one allowed by Pauli's exclusion principle. The anisotropy of the electric dipolar interaction selects the instability in the $p_{z}$-channel, which is slightly hybridized with other odd partial wave channels ${ }^{20-27}$. For two-component cases, the dipolar interaction leads to anisotropic spin-triplet pairing, and its orbital partial wave is again in the $p_{z}$-channel ${ }^{28-31}$. The triplet pairing competes with the singlet pairing in the hybridized $s+d_{r^{2}}-3 z^{2}$-channel. The mixing between the singlet and triplet pairings has a relative phase $\pm \frac{\pi}{2}$, which leads to a novel time-reversal symmetry breaking Cooper pairing state ${ }^{29}$

An important recent experimental progress is the laser cooling and trapping of magnetic dipolar fermions of ${ }^{161} \mathrm{Dy}$ and ${ }^{163} \mathrm{Dy}$ with large atomic magnetic moments $\left(10 \mu_{B}\right)^{1,2}$. There are important differences between magnetic and electric dipolar interactions. Electric dipole moments are essentially non-quantized classic vectors from the mixing between different rotational eigenstates with opposite parities, which are induced by external electric fields ${ }^{3,4}$, thus electric dipoles are frozen. In the absence of external fields, even though at each instant of time there is a dipole moment of the heteronuclear molecule, it is averaged to zero at a long time scale. In contrast, magnetic dipole moments of atoms are intrinsic, proportional to their hyper-fine spins with a Lande factor. Unpolarized magnetic dipolar Fermi systems are available, in which dipoles are defrozen as non-commutative quantum mechanical operators, thus lead to richer quantum spin physics of dipolar interactions. Furthermore, the magnetic dipolar interaction is actually isotropic in the unpolarized systems. It is invariant under simultaneous spinorbit rotations but not separate spin or orbit rotations. This spin-orbit coupling is different from usual single particle one, but an interaction effect. It plays an important role in the Fermi liquid properties such as the unconventional magnetic states and ferro-nematic states predicted by Fregoso et $\mathrm{al}^{18,19}$.

It is natural to expect that magnetic dipolar interaction brings novel pairing symmetries not studied in condensed matter systems before. The systems of ${ }^{161} \mathrm{Dy}$ and ${ }^{163} \mathrm{Dy}$ are with a very large hyperfine spin of $F=\frac{21}{2}$, thus their Cooper pairing problem is expected to be very challenging. As a first step, we study the simplest case of spin- $\frac{1}{2}$, and find that the magnetic dipolar interaction provides a novel and robust mechanism to the $p$ wave $(L=1)$ spin triplet $(S=1)$ Cooper pairing to the first order of interaction strength, which comes from the 
attractive part of the magnetic dipolar interaction. In comparison, the $p$-wave triplet pairing in usual condensed matter systems, such as ${ }^{3} \mathrm{He}^{32-34}$, is due to the spin-fluctuation mechanism, which is at the second order of interaction strength (see Refs. ${ }^{35,36}$ for reviews). This mechanism is based on strong ferromagnetic tendency from the repulsive part of the ${ }^{3} \mathrm{He}-{ }^{3} \mathrm{He}$ interactions. Furthermore, the $p$-wave triplet Cooper pairing symmetry patterns in magnetic dipolar systems are novel, which do not appear in ${ }^{3} \mathrm{He}$. The orbital and spin angular momenta of the Cooper pair are entangled into the total angular momentum $J=1$, which is denoted as the $J$-triplet channel below. In contrast, in the ${ }^{3} \mathrm{He}-B$ phase ${ }^{33}, L$ and $S$ are combined into $J$ $=0$; and in the ${ }^{3} \mathrm{He}-A$ phase, $L$ and $S$ are decoupled and $J$ is not welldefined $^{32,34}$. There are two competing pairing possibilities in this $J$ triplet channel with different values of $J_{z}$ : the helical polar state $\left(J_{z}=\right.$ $0)$ preserving time reversal (TR) symmetry, and the axial state $\left(J_{z}=\right.$ $\pm 1)$ breaking TR symmetry. The helical polar state has point nodes and gapless Dirac spectra, which is a time-reversal invariant generalization of the ${ }^{3} \mathrm{He}-A$ phase with entangled spin and orbital degrees of freedom. In addition to usual phonon modes, its Goldstone modes contain the total angular momentum wave as entangled spin-orbital modes.

\section{Results}

We begin with the magnetic dipolar interaction between spin- $\frac{1}{2}$ fermions

$$
V_{\alpha \beta, \beta^{\prime} \alpha^{\prime}}(\vec{r})=\frac{\mu^{2}}{r^{3}}\left\{\vec{S}_{\alpha \alpha^{\prime}} \cdot \vec{S}_{\beta \beta^{\prime}}-3\left(\vec{S}_{\alpha \alpha^{\prime}} \cdot \hat{r}\right)\left(\vec{S}_{\beta \beta^{\prime}} \cdot \hat{r}\right)\right\}
$$

where $\vec{r}$ is the relative displacement vector between two fermions; $\mu$ is the magnitude of the magnetic moment. Such an interaction is invariant under the combined $S U(2)$ spin rotation and $S O(3)$ space rotation. In other words, orbital angular momentum $\vec{L}$ and spin $\vec{S}$ are not separately conserved, but the total angular momentum $\vec{J}=\vec{L}+\vec{S}$ remains conserved. Its Fourier transformation reads ${ }^{19}$

$$
V_{\alpha \beta ; \beta^{\prime} \alpha^{\prime}}(\vec{q})=\frac{4 \pi}{3} \mu^{2}\left\{3\left(\vec{S}_{\alpha \alpha^{\prime}} \cdot \hat{q}\right)\left(\vec{S}_{\beta \beta^{\prime}} \cdot \hat{q}\right)-\vec{S}_{\alpha \alpha^{\prime}} \cdot \vec{S}_{\beta \beta^{\prime}}\right\} .
$$

The Hamiltonian in the second quantization form is written as

$$
\begin{aligned}
H= & \sum_{\vec{k}, \alpha}\left[\epsilon(\vec{k})-\mu_{c}\right] c_{\alpha}^{\dagger}(\vec{k}) c_{\alpha}(\vec{k}) \\
& +\frac{1}{2 V} \sum_{\vec{k}, \vec{k}^{\prime}, \vec{q}} V_{\alpha \beta ; \beta^{\prime} \alpha^{\prime}}\left(\vec{k}-\vec{k}^{\prime}\right) P_{\alpha \beta}^{\dagger}(\vec{k} ; \vec{q}) P_{\beta^{\prime} \alpha^{\prime}}\left(\vec{k}^{\prime} ; \vec{q}\right),
\end{aligned}
$$

where $\epsilon(\vec{k})=\hbar k^{2} /(2 m) ; \quad \mu_{c} \quad$ is the chemical potential; $P_{\beta^{\prime} \alpha^{\prime}}(\vec{k} ; \vec{q})=c_{\beta^{\prime}}(-\vec{k}+\vec{q}) c_{\alpha^{\prime}}(\vec{k}+\vec{q})$ is the pairing operator; the Greek indices $\alpha, \beta, \alpha^{\prime}$ and $\beta^{\prime}$ refer to $\uparrow$ and $\downarrow ; V$ is the volume of the system. We define a dimensionless parameter characterizing the interaction strength as the ratio between the characteristic interaction energy and the Fermi energy: $\lambda \equiv E_{\text {int }} / E_{F}=\frac{2}{3} \frac{\mu^{2} m k_{f}}{\pi^{2} \hbar^{2}}$.

We next study the symmetry of the Cooper pairing in the presence of Fermi surface, i.e., in the weak coupling theory. An important feature of the magnetic dipolar interaction in Eq. (1) is that it vanishes in the total spin singlet channel. Thus, we only need to study the triplet pairing in odd orbital partial wave channels. Considering uniform pairing states at the mean-field level, we set $\vec{q}=0$ in Eq. (3), and define triplet pairing operators $P_{s}(\vec{k})$, which are eigen-operators of $\vec{S}_{1 z}+\vec{S}_{2 z}$ with eigenvalues $s_{z}=0, \pm 1$, respectively. More explicitly, they $\quad$ are $\quad P_{0}(\vec{k})=\frac{1}{\sqrt{2}}\left[P_{\uparrow \downarrow}(\vec{k})+P_{\downarrow \uparrow}(\vec{k})\right], \quad P_{1}(\vec{k})=P_{\uparrow \uparrow}(\vec{k})$, $P_{-1}(\vec{k})=P_{\downarrow \downarrow}(\vec{k})$. The pairing interaction of Eq. (3) reduces to

$$
H_{\text {pair }}=\frac{1}{2 V} \sum_{\vec{k}, \vec{k}^{\prime}, s_{z} s_{z}^{\prime}}\left\{V_{s_{z} s_{z}^{\prime}}^{T}\left(\vec{k} ; \vec{k}^{\prime}\right) P_{s_{z}}^{\dagger}(\vec{k}) P_{s_{z}^{\prime}}\left(\vec{k}^{\prime}\right)\right\}
$$

where

$$
\begin{aligned}
V_{s_{z} s_{z}^{\prime}}^{T}\left(\vec{k} ; \vec{k}^{\prime}\right)= & \frac{1}{2} \sum_{\alpha \beta \beta^{\prime} \alpha^{\prime}}\left\langle 1 s_{z} \mid \frac{1}{2} \alpha \frac{1}{2} \beta\right\rangle\left\langle 1 s_{z}^{\prime} \mid \frac{1}{2} \alpha^{\prime} \frac{1}{2} \beta^{\prime}\right\rangle * \\
& \left\{V_{\alpha \beta, \beta^{\prime} \alpha^{\prime}}\left(\vec{k}-\vec{k}^{\prime}\right)-V_{\alpha \beta, \beta^{\prime} \alpha^{\prime}}\left(\vec{k}+\vec{k}^{\prime}\right)\right\} .
\end{aligned}
$$

$\left\langle 1 s_{z} \mid \frac{1}{2} \alpha \frac{1}{2} \beta\right\rangle$ is the Clebsch-Gordan coefficient for two spin- $\frac{1}{2}$ states to form the spin triplet; and $V_{s_{z} s_{z}^{\prime}}\left(\vec{k} ; \vec{k}^{\prime}\right)$ is an odd function of both $\vec{k}$ and $\vec{k}^{\prime}$.

The decoupled mean-field Hamiltonian reads

$$
H_{m f}=\frac{1}{2 V} \sum_{\vec{k}}^{\prime} \Psi^{\dagger}(\vec{k})\left(\begin{array}{cc}
\xi(\vec{k}) I & \Delta_{\alpha \beta}(\vec{k}) \\
\Delta_{\beta \alpha}^{*}(\vec{k}) & -\xi(\vec{k}) I
\end{array}\right) \Psi(\vec{k}),
$$

where we only sum over half of the momentum space; $\xi(\vec{k})=\epsilon(\vec{k})-\mu_{c h}$ and $\mu_{c h} \quad$ is the chemical potential; $\Psi(\vec{k})=\left(c_{\uparrow}(\vec{k}), c_{\downarrow}(\vec{k}), c_{\uparrow}^{\dagger}(-\vec{k}), c_{\downarrow}^{\dagger}(-\vec{k})\right)^{T} ; \Delta_{\alpha \beta}$ is defined as $\Delta_{\alpha \beta}=\sum s_{z}\left\langle 1 s_{z} \mid \frac{1}{2} \alpha \frac{1}{2} \beta\right\rangle * \Delta_{s_{z}}$. $\Delta_{s_{z}}$ satisfies the mean-field gap function as

$$
\begin{aligned}
\Delta_{s_{z}}(\vec{k}) & =\frac{1}{V} \sum_{\vec{k}^{\prime},_{z}^{\prime}} V_{s_{z} s_{z}^{\prime}}^{T}\left(\vec{k} ; \vec{k}^{\prime}\right)\left\langle\left|P_{s_{z}^{\prime}}\left(\vec{k}^{\prime}\right)\right|\right\rangle \\
& =-\int \frac{d^{3} k^{\prime}}{(2 \pi)^{3}} V_{s_{z} s_{z}^{\prime}}^{T}\left(\vec{k} ; \vec{k}^{\prime}\right)\left[K\left(\vec{k}^{\prime}\right)-\frac{1}{2 \epsilon_{k}}\right] \Delta_{s_{z}^{\prime}}\left(\vec{k}^{\prime}\right),
\end{aligned}
$$

where $K\left(\vec{k}^{\prime}\right)=\tanh \left[\frac{\beta}{2} E_{i}\left(\vec{k}^{\prime}\right)\right] /\left[2 E_{i}\left(\vec{k}^{\prime}\right)\right]$. The integral in Eq. (7) is already normalized following the standard procedure ${ }^{20}$. For simplicity, we use the Born approximation in Eq. (7) by employing the bare interaction potential rather than the fully renormalized $T$ matrix, which applies in the dilute limit of weak interactions. The pairing symmetry, on which we are interested below, does not depend on the details that how the integral of Eq. (7) is regularized in momentum space. The Bogoliubov quasiparticle spectra become $E_{1,2}(\vec{k})=\sqrt{\xi_{k}^{2}+\lambda_{1,2}^{2}(\vec{k})}$, where $\lambda_{1,2}^{2}(\vec{k})$ are the eigenvalues of the positive-definite Hermitian matrix $\Delta^{\dagger}(\vec{k}) \Delta(\vec{k})$. The free energy can be calculated as

$$
\begin{aligned}
F= & -\frac{2}{\beta} \sum_{\vec{k}, i=1,2} \ln \left[2 \cosh \frac{\beta E_{\vec{k}, i}}{2}\right]- \\
& \frac{1}{2 V} \sum_{\vec{k}, \vec{k}^{\prime}, s_{z}, s_{z}^{\prime}}\left\{\Delta_{s_{z}}^{*}(\vec{k}) V_{s_{z} s_{z}^{\prime}}^{T,-1}\left(\vec{k} ; \vec{k}^{\prime}\right) \Delta_{s_{z}^{\prime}}(\vec{k})\right\},
\end{aligned}
$$

where $V_{s_{z} s_{z}^{\prime}}^{T,-1}\left(\vec{k} ; \vec{k}^{\prime}\right)$ is the inverse of the interaction matrix defined as

$$
\frac{1}{V} \sum_{\vec{k}^{\prime}, s_{z}^{\prime}} V_{s_{z}, s_{z}^{\prime}}^{T}\left(\vec{k}, \vec{k}^{\prime}\right) V_{s_{z}^{\prime}, s_{z}^{\prime \prime}}^{T,-1}\left(\vec{k}^{\prime} ; \vec{k}^{\prime \prime}\right)=\Delta_{\vec{k}, \vec{k}^{\prime \prime}} \Delta_{s_{z}, s_{z}^{\prime \prime}} .
$$

We next linearize Eq. (7) around $T_{c}$ and perform the partial wave analysis to determine the dominant pairing channel. Since the total angular momentum is conserved, we can use $J$ to classify the 
eigen-gap functions denoted as $\phi_{s_{z}}^{a, J J_{z}}(\vec{k})$. The index $a$ is used to distinguish different channels sharing the same value of $J . \phi_{s_{z}}^{a, J J_{z}}(\vec{k})$ satisfies

$$
N_{0} \int \frac{d \Omega_{k^{\prime}}}{4 \pi} V_{s_{z} s_{z}^{\prime}}^{T}\left(\vec{k} ; \vec{k}^{\prime}\right) \phi_{s_{z}^{\prime}}^{a ; J J_{z}}\left(\vec{k}^{\prime}\right)=w_{J}^{a} \phi_{s_{z}}^{a ; J J_{z}}(\vec{k}),
$$

where $N_{0}=\frac{m k_{f}}{\pi^{2} \hbar^{2}}$ is the density of state at the Fermi surface; $w_{j}^{a}$ are dimensionless eigenvalues; $\vec{k}, \vec{k}^{\prime}$ are at the Fermi surface. Then Eq. (7) is linearized into a set of decoupled equations

$$
\phi^{a ; J J_{z}}\left\{1+w_{J}^{a}\left[\ln \left(2 e^{\gamma} \bar{\Omega}\right) /\left(\pi k_{B} T\right)\right]\right\}=0,
$$

where $\bar{\Omega}$ is an energy scale at the order of the Fermi energy playing the role of energy cut-off from the Fermi surface.

The decomposition of $V_{s_{z} s_{z}^{\prime}}^{T}\left(\vec{k} ; \overrightarrow{k^{\prime}}\right)$ into spherical harmonics can be formulated as

$$
\frac{N_{0}}{4 \pi} V_{s_{z} s_{z}^{\prime}}^{T}\left(\vec{k} ; \vec{k}^{\prime}\right)=\sum_{L m, L^{\prime} m^{\prime}} V_{L m s_{z} ; L^{\prime} m^{\prime} s_{z}^{\prime}} Y_{L m}^{*}\left(\Omega_{k}\right) Y_{L^{\prime} m^{\prime}}\left(\Omega_{\overrightarrow{k^{\prime}}}\right)
$$

where $L=L^{\prime}$ or $L=L^{\prime} \pm 2$, and $L, L^{\prime}$ are odd numbers. The expressions of the dimensionless matrix elements $V_{L m s_{z} ; L^{\prime} m^{\prime} s_{z}^{\prime}}$ are lengthy and will be presented elsewhere. By diagonalizing this matrix, we find that the most negative eigenvalues is $w^{J=1}=-3 \pi \lambda / 4$ lying in the channel with $J=L=1$. All other negative eigenvalues are significantly smaller. Therefore, dominate pairing symmetry is identified as the $J$-triplet channel with $L=S=1$ in the weak coupling theory. Following the standard method in Ref. ${ }^{20}$, the transition temperature $T_{c}$ is expressed as $T_{c} \approx \frac{2 e^{\gamma} \bar{\Omega}}{\pi} e^{-\frac{1}{\left|w^{\prime}=1\right|}}$. For a rough estimation of the order of magnitude of $T_{\mathcal{c}}$, we set the prefactor in the expression of $T_{c}$ as $E_{f}$.

In order to understand why the $J$-triplet channel is selected by the magnetic dipolar interaction, we present a heuristic picture based on a two-body pairing problem in real space. Dipolar interaction has a characteristic length scale $a_{d p}=m \mu^{2} / \hbar^{2}$ at which the kinetic energy scale equals the interaction energy scale. We are not interested in solving the radial equation but focus on the symmetry properties of the angular solution, thus, the distance between two spins is taken fixed at $a_{d p}$. We consider the lowest partial-wave, $p$-wave, channel with $L=1$. The $3 \times 3=9$ states $(L=S=1)$ are classified into three sectors of $J=0,1$ and 2 . In each channel of $J$, the interaction energies are diagonalized as

$$
E_{0}=E_{d p}, \quad E_{1}=-\frac{1}{2} E_{d p}, \quad E_{2}=\frac{1}{10} E_{d p},
$$

respectively, where $E_{d p}=\mu^{2} / a_{d p}^{3}$. Only the total angular momentum triplet sector with $J=1$ supports bound states, thus is the dominant pairing channel and is consistent with the pairing symmetry in the weak-coupling theory.

This two-body picture applies in the strong coupling limit. Although a complete study of the strong coupling problem is beyond the scope of this paper, this result provides an intuitive picture to understand pairing symmetry in the $J$-triplet sector from spin configurations. We define that $\chi_{\mu}$ and $p_{\mu}(\hat{\Omega})$ are eigenstates with eigenvalues zero for operators $\hat{e}_{\mu} \cdot\left(\vec{S}_{1}+\vec{S}_{2}\right)$ and $\hat{e}_{\mu} \cdot \vec{L}(\mu=x, y, z)$, which are the total spin and orbital angular momenta projected along the $e_{\mu}$-direction. The $J$-triplet sector states are $\phi_{\mu}(\Omega)=$ $\frac{1}{\sqrt{2}} \epsilon_{\mu \nu \lambda} \chi_{\nu} p_{\lambda}(\Omega)$ with $\phi_{\mu}$ satisfying $\left(\hat{e}_{\mu} \cdot \vec{J}\right) \phi_{\mu}=0$. For example,

$$
\begin{aligned}
\phi_{z}(\hat{\Omega}) & =\frac{1}{\sqrt{2}}\left[\chi_{x} p_{y}(\hat{\Omega})-\chi_{y} p_{x}(\hat{\Omega})\right] \\
& =\sqrt{\frac{3}{2}} \sin \theta\left\{\left|\alpha_{\hat{e}_{\rho}}\right\rangle_{1}\left|\alpha_{\hat{e}_{\rho}}\right\rangle_{2}+\left|\beta_{\hat{e}_{\rho}}\right\rangle_{1}\left|\beta_{\hat{e}_{\rho}}\right\rangle_{2}\right\},
\end{aligned}
$$
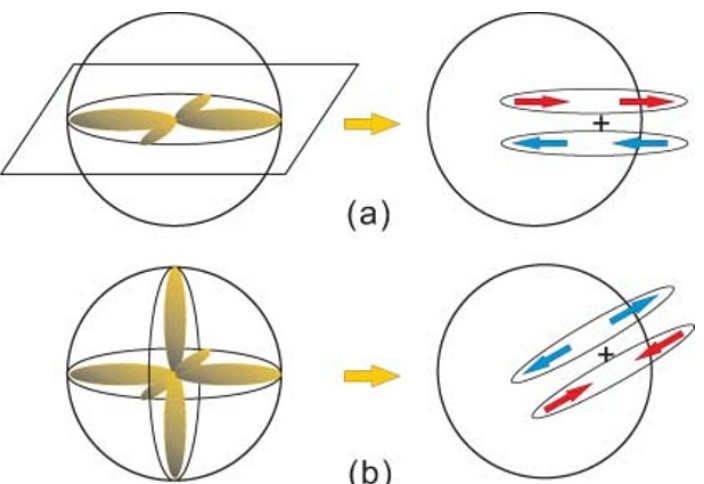

Figure $1 \mid$ The spin configurations of the two-body states with a) $J=1$ and $j_{z}=0$ and b) $J=j_{z}=0$. The interactions are attractive in a) but repulsive in $b$ ).

where $\hat{e}_{\rho}=\hat{x} \cos \phi+\hat{y} \sin \phi$ and $\left|\alpha_{e_{\rho}}\right\rangle$ and $\left|\beta_{e_{\rho}}\right\rangle$ are eigenstates of $\hat{e}_{\rho} \cdot \vec{\sigma}$ with eigenvalues of \pm 1 . As depicted in Fig. $1 \mathrm{~A}$, along the equator where $\phi_{z}$ has the largest weight, two spins are parallel and along $\hat{r}$, thus the interaction is dominated by attraction. On the other hand, the eigenstate of $J=0$ reads

$$
\phi_{0}(\Omega)=\chi_{\mu} p_{\mu}(\Omega)=\frac{1}{\sqrt{2}}\left\{\left|\alpha_{\Omega}\right\rangle_{1}\left|\beta_{\Omega}\right\rangle_{2}+\left|\beta_{\Omega}\right\rangle_{1}\left|\alpha_{\Omega}\right\rangle_{2}\right\}
$$

where $\left|\alpha_{\Omega}\right\rangle$ and $\left|\beta_{\Omega}\right\rangle$ are eigenstates of $\hat{\Omega} \cdot \vec{\sigma}$ with eigenvalues \pm 1 . As shown in Fig. $1 \mathrm{~B}$, along any direction of $\hat{\Omega}$, two spins are anti-parallel and longitudinal, thus the interaction is repulsive.

Let us come back to momentum space and study the competition between three paring branches in the J-triplet channel under the Ginzburg-Landau (GL) framework. We define

$$
\begin{aligned}
& \Delta_{x}(\vec{k})=\frac{1}{\sqrt{2}}\left[-\Delta_{1}(\vec{k})+\Delta_{-1}(\vec{k})\right], \\
& \Delta_{y}(\vec{k})=\frac{i}{\sqrt{2}}\left[\Delta_{1}(\vec{k})+\Delta_{-1}(\vec{k})\right], \quad \Delta_{z}(\vec{k})=\Delta_{0}(\vec{k}) .
\end{aligned}
$$

The bulk pairing order parameters are defined as $\Delta_{\mu}=\frac{1}{V} \sum_{k}$ $\hat{k}_{\mu} \Delta_{\mu}(\vec{k})$, where no summation over $\mu$ is assumed. We define pairing parameters and their real and imaginary parts as the following 3vectors $\vec{\Delta}=\left(\Delta_{x}, \Delta_{y}, \Delta_{z}\right)$. The GL free energy is constructed to maintain the $U(1)$ and $S O(3)$ rotational symmetry as

$$
F=\alpha \vec{\Delta}^{*} \cdot \vec{\Delta}+\gamma_{1}\left|\vec{\Delta}^{*} \cdot \vec{\Delta}\right|^{2}+\gamma_{2}\left|\vec{\Delta}^{*} \times \vec{\Delta}\right|^{2},
$$

where

$$
\alpha=N_{0} \ln \left(\frac{T}{T_{c}}\right) .
$$

The sign of $\gamma_{2}$ determines two different pairing structures: $\operatorname{Re} \vec{\Delta} \| \operatorname{Im} \vec{\Delta}$ at $\gamma_{2}>0$, and $\operatorname{Re} \vec{\Delta} \perp \operatorname{Im} \vec{\Delta}$ at $\gamma_{2}<0$, respectively. Using the analogy of the spinor condensation of spin-1 bosons, the former is the polar pairing state and the latter is the axial pairing state s7-40. $^{37}$.

For the polar pairing state, the order parameter configuration can be conveniently denoted as $\vec{\Delta}=e^{i \phi}|\Delta| \hat{z}$ up to a $U(1)$ phase and $S O(3)-$ rotation. This pairing carries the quantum number $J_{z}=0$. The pairing matrix $\left.\Delta_{\alpha \beta}^{p l}=\frac{1}{2}|\Delta|\left[k_{y} \sigma_{1}-k_{x} \sigma_{2}\right) i \sigma_{2}\right]_{\alpha \beta}$ reads

$$
\Delta_{\alpha \beta}^{p l}=\frac{1}{2}|\Delta|\left[\begin{array}{cc}
-\left(\hat{k}_{y}+i \hat{k}_{x}\right) & 0 \\
0 & \hat{k}_{y}-i \hat{k}_{x}
\end{array}\right] .
$$


It equivalents to a superposition of $p_{x} \mp i p_{y}$ orbital configurations for spin- $\uparrow \uparrow(\downarrow \downarrow)$ pairs, respectively. Thus, this pairing state is helical. It is a unitary pairing state because $\hat{\Delta}^{\dagger} \hat{\Delta}$ is proportional to a $2 \times 2$ identity matrix. The Bogoliubov quasiparticle spectra are degenerate for two different spin configurations as $E_{k, \alpha}^{p l}=\sqrt{\xi_{k}^{2}+\left|\Delta^{p l}(\vec{k})\right|^{2}}$ with the anisotropic gap function $\left|\Delta^{p l}(\vec{k})\right|^{2}=\frac{1}{4}|\Delta|^{2} \sin ^{2} \theta_{k}$ depicted in Fig. 2. They exhibit Dirac cones at north and south poles with opposite chiralities for two spin configurations.

Similarly, the order parameter configuration in the axial pairing state can be chosen as $\vec{\Delta}=\frac{1}{\sqrt{2}} e^{i \phi}|\Delta|\left(\hat{e}_{x}+i \hat{e}_{y}\right)$ up to the symmetry transformation. This state carries the quantum number of $J_{z}=1$. The pairing matrix $\Delta_{\alpha \beta}^{a x}=\frac{1}{2 \sqrt{2}}|\Delta|\left\{\left[\hat{k}_{z}\left(\sigma_{1}+i \sigma_{2}\right)+\sigma_{z}\left(\hat{k}_{x}+i \hat{k}_{y}\right] i \sigma_{2}\right\}_{\alpha \beta}\right.$
takes the form

$$
\Delta_{\alpha \beta}^{a x}=\frac{\sqrt{2}}{2}|\Delta|\left[\begin{array}{cc}
\hat{k}_{z} & \frac{1}{2}\left(\hat{k}_{x}+i \hat{k}_{y}\right) \\
\frac{1}{2}\left(\hat{k}_{x}-i \hat{k}_{y}\right) & 0
\end{array}\right] .
$$

This is a non-unitary pairing state since $\Delta^{\dagger} \Delta=$ $|\Delta|^{2}\left[\frac{1}{2}\left(1+\hat{k}_{z}^{2}\right)+\hat{k}_{z}(\hat{k} \cdot \vec{\sigma})\right]$. The Bogoliubov quasiparticle spectra have two non-degenerate branches with anisotropic dispersion relations as $E_{1,2}^{a x}(\vec{k})=\sqrt{\xi_{k}^{2}+\left|\Delta_{ \pm}^{a x}(\vec{k})\right|^{2}}$. The angular gap distribution $\left|\Delta_{ \pm}^{a x}(\vec{k})\right|^{2}=\frac{1}{8}|\Delta|^{2}\left(1 \pm \cos \theta_{k}\right)^{2}$ is depicted in Fig. 2. Each of branch 1 and 2 exhibits one node at north pole and south pole, respectively. Around the nodal region, the dispersion simplifies into $E_{1,2}(\vec{k})=\sqrt{v_{f}^{2}\left(k_{z} \mp k_{f}\right)^{2}+\frac{1}{32}|\Delta|^{2}\left(k_{\|} / k_{f}\right)^{4}}$, which is quadratic in the transverse momentum $k_{\|}=\sqrt{k_{x}^{2}+k_{y}^{2}}$.

At the mean-field level, the helical polar pairing state is more stable than the axial state. Actually, this conclusion is not so obvious as in the case of ${ }^{3} \mathrm{He}-\mathrm{B}$ phase, where the isotropic gap function is the most stable among all the possible gap functions ${ }^{33}$. Here, the gap functions are anisotropic in both the polar and helical pairing phases. We need to compare them by calculating their free energies in Eq. (8). The second term contributes the same to both pairing phases. Thus, the first term determines the difference in free energies. Let us define the ratio between angular integrals of the free energy kernels in Eq. (8) of the two phases as

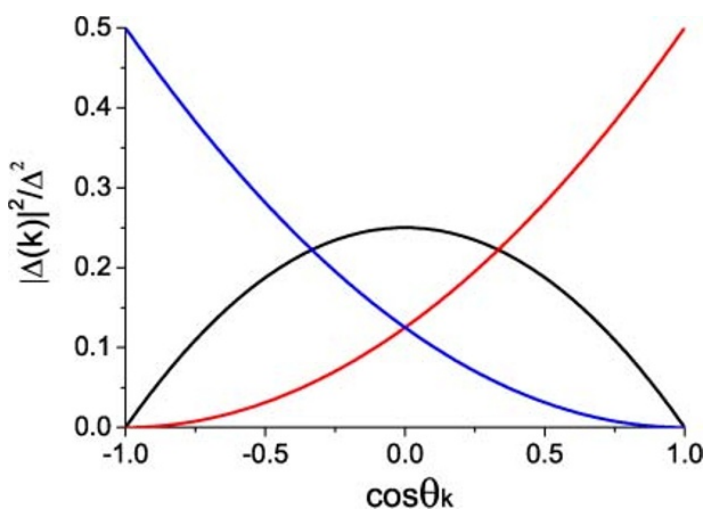

Figure $2 \mid$ The angular distribution of the gap function $|\Delta(\vec{k})|^{2}$ v.s. $\cos \theta_{k}$ in the helical polar pairing state (the red line) and the axial pairing state (the black line).

$$
y\left(\lambda_{1}, \lambda_{2}\right)=\frac{\int d \Omega_{k} 2 \ln \left[2 \cosh \frac{\beta}{2} \sqrt{\xi_{k}^{2}+\left|\Delta^{p l}(\vec{k})\right|^{2}}\right]}{\int d \Omega_{k} \sum_{ \pm} \ln \left[2 \cosh \frac{\beta}{2} \sqrt{\xi_{k}^{2}+\left|\Delta_{ \pm}^{a x}(\vec{k})\right|^{2}}\right]}
$$

where $\lambda_{1}=\frac{1}{\beta|\Delta|}, \lambda_{2}=\frac{1}{\beta|\xi k|} \cdot y\left(\lambda_{1}, \lambda_{2}\right)$ is numerically plotted in Fig. 3 . For arbitrary values of $\beta, \xi_{k}$, and $|\Delta|, y$ is always larger than 1 . Therefore, the polar state is favored more than the axial state. This can be understood from the convexity of the nonlinear term in Eq. (8), which favors isotropic angular distributions of $|\Delta(\vec{k})|^{2}{ }^{42}$. Although neither gap function of these two states is absolutely isotropic as in the ${ }^{3} \mathrm{He}-\mathrm{B}$ phase, the polar gap function is more isotropic from Fig. 2 and thus is favored. However, we need to bear in mind that we cannot rule out the possibility that certain strong coupling effects can stabilize the axial state. In fact, the ${ }^{3} \mathrm{He}-\mathrm{A}$ phase can be stabilized under the spin feedback mechanism ${ }^{35}$, which is a higher order effect in terms of interaction strength.

Next we discuss the classification of Goldstone modes and vortices in these two states. In the helical polar state, the remaining symmetries are $S O_{J}(2) \times Z_{2}$ as well as parity and time-reversal (TR), where $Z_{2}$ means the combined operation of rotation $\pi$ around any axis in the $x y$-plane and a flip of the pairing phase by $\pi$. The Goldstone manifold is

$$
\left[S O_{J}(3) \times U_{c}(1)\right] /\left[S O_{J}(2) \otimes Z_{2}\right]=\left[S_{J}^{2} \times U_{c}(1)\right] / Z_{2} .
$$

The Goldstone modes include the phase phonon mode and two branches of spin-orbital modes. Vortices in this phase can be classified into the usual integer vortices in the phase sector and halfquantum vortices combined with $\pi$-disclination of the orientation of $\vec{\Delta}$. In the axial state, the rotation around $z$-axis generates a shift of the pairing phase, which can be canceled by a $U_{c}(1)$ transformation, thus, the remaining symmetry is $S_{J_{z}-\phi}(2)$. The Goldstone manifold is $S^{2} \times U_{c}(1)$. Only integer vortices exist.

\section{Discussions}

In summary, we have found that the magnetic dipolar interaction provides a robust mechanism at first order in the interaction strength for a novel $p$-wave $(L=1)$ spin triplet $(S=1)$ Cooper pairing state, in which the total angular momentum of the Cooper pair is $J=1$. This is a novel pairing pattern which does not appear in ${ }^{3} \mathrm{He}$, and, to our knowledge, neither in any other condensed matter systems. These pairing states include the TR invariant helical polar pairing state and

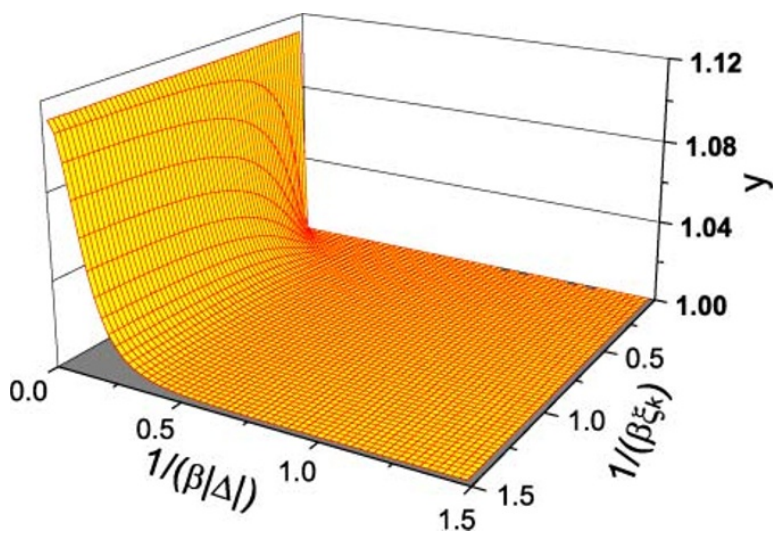

Figure $3 \mid$ The ratio of the angular integrals of the free energy kernels $y\left(\frac{1}{\boldsymbol{\beta}|\Delta|}, \frac{1}{\boldsymbol{\beta}|\xi|}\right)$, which is always larger than 1 . This means that the polar pairing is favored at the mean-field level. 
the TR breaking axial pairing state, both of which are distinct from the familiar ${ }^{3} \mathrm{He}-A$ and $B$ phases.

Many interesting questions are open for further exploration, including the topological properties of these pairing states, vortices, spin textures, and spectra of collective excitations. The above theory only applies for spin- $-\frac{1}{2}$ systems, in which the magnetic dipolar interaction is too small. For the pairing symmetry in a magnetic dipolar system with a large spin $S$, our preliminary results show that the basic features of the $J$-triplet pairing remains. The spins of two fermions are parallel forming $S_{t o t}=2 S$ with orbital partial-wave $L=1$, and the total $J=2 S$. In the current experiments in Ref. ${ }^{41}$, the highest attainable density reaches $4 \times 10^{13} \mathrm{~cm}^{-1}$ for ${ }^{161}$ Dy atoms with $S=\frac{21}{2}$. The corresponding dipolar energy is $E_{\text {int }} \approx 2 \mathrm{nK}$ and the Fermi energy for unpolarized gases $E_{f} \approx 13.6 \mathrm{nK}$, and thus $\lambda=E_{\text {int }} / E_{f} \approx 0.15$. If we use the same formula of $w^{J=1}$ above for an estimation of the most negative eigenvalue, we arrive at $T_{c} / T_{f} \approx 0.06$, which means that $T_{c} \approx 0.8 \mathrm{nK}$. Although it is still slightly below the lower limit of the accessible temperature in current experiments, we expect that further increase of fermions density, say, in optical lattices will greatly increase $T_{c}$.

\section{Method}

We have used the methods of the symmetry analysis, strong coupling analysis, meanfield theory, partial-wave analysis, and the Ginzburg-Landau free energy, which have been explained in Sec. I.

1. Lu, M., Youn, S. H. \& Lev, B. L. Trapping ultracold dysprosium: a highly magnetic gas for dipolar physics. Phys. Rev. Lett. 104, 63001 (2010).

2. Youn, S. H., Lu, M., Ray, U. \& Lev, B. L. Dysprosium magneto-optical traps. Phys. Rev. A 82, 043425 (2010).

3. Ospelkaus, S. et al. Ultracold polar molecules near quantum degeneracy. Faraday Discuss. 142, 351 (2009).

4. Ni, K. K. et al. A high phase-space-density gas of polar molecules. Science 322, 231 (2008)

5. Griesmaier, A., Werner, J., Hensler, S., Stuhler, J. \& Pfau, T. Bose-einstein condensation of chromium. Phys. Rev. Lett. 94, 160401 (2005).

6. McClelland, J. J. \& Hanssen, J. L. Laser cooling without repumping: a magnetooptical trap for erbium atoms. Phys. Rev. Lett. 96, 143005 (2006).

7. Aikawa, K. et al. Bose-Einstein Condensation of Erbium. arXiv:1204.1725 (2012).

8. Koch, T., Lahaye, T., Metz, J., Fröhlich, B., Griesmaier, A. \& Pfau, T. Stabilization of a purely dipolar quantum gas against collapse. Nat. Phys. 4, 218-222 (2008)

9. Lahaye, T., Menotti, C., Santos, L., Lewenstein, M. \& Pfau, T. The physics of dipolar bosonic quantum gases. Rep. Prog. Phys. 72, 126401 (2009).

10. Lahaye, T., Metz, J., Koch, T., Fröhlich, B., Griesmaier, A. \& Pfau, T. A purely dipolar quantum gas. 21st International Conference on Atomic Physics, 160. World Scientific, (2009).

11. Menotti, C., Lewenstein, M., Lahaye, T. \& Pfau, T. Dipolar interaction in ultracold atomic gases. Dynamics and Thermodynamics of Systems with Long Range Interactions: Theory and Experiments, vol. 970, 332-361 (2008).

12. Sogo, T., He, L., Miyakawa, T., Yi, S., Lu, H. \& Pu, H. Dynamical properties of dipolar fermi gases. New J. Phys. 11, 055017 (2009)

13. Miyakawa, T., Sogo, T. \& Pu, H. Phase-space deformation of a trapped dipolar fermi gas. Phys. Rev. A 77, 061603 (2008).

14. Ronen, S. \& Bohn, J. L. Zero sound in dipolar fermi gases. Phys. Rev. A 81, 033601 (2010)

15. Chan, C. K., Wu, C., Lee, W. C. \& Sarma, S. D. Anisotropic-fermi-liquid theory of ultracold fermionic polar molecules: Landau parameters and collective modes. Phys. Rev. A 81, 023602 (2010)

16. Fregoso, B. M., Sun, K., Fradkin, E. \& Lev, B. L. Biaxial nematic phases in ultracold dipolar fermi gases. New J. Phys. 11, 103003 (2009).

17. Lin, C., Zhao, E. \& Liu, W. V. Liquid crystal phases of ultracold dipolar fermions on a lattice. Phys. Rev. B 81, 045115 (2010).

18. Fregoso, B. M. \& Fradkin, E. Unconventional magnetism in imbalanced fermi systems with magnetic dipolar interactions. Phys. Rev. B 81, 214443 (2010).
19. Fregoso, B. M. \& Fradkin, E. Ferronematic ground state of the dilute dipolar fermi gas. Phys. Rev. Lett. 103, 205301 (2009).

20. Baranov, M. A., Mar'enko, M. S., Rychkov, V. S. \& Shlyapnikov, G. V. Superfluid pairing in a polarized dipolar fermi gas. Phys. Rev. A 66, 013606 (2002)

21. Baranov, M. A., Dobrek, L. \& Lewenstein, M. Superfluidity of trapped dipolar fermi gases. Phys. Rev. Lett. 92, 250403 (2004).

22. Baranov, M. A. Theoretical progress in many-body physics with ultracold dipolar gases. Physics Reports 464, 71-111 (2008)

23. You, L. \& Marinescu, M. Prospects for p-wave paired bardeen-cooper-schrieffer states of fermionic atoms. Phys. Rev. A 60, 2324 (1999).

24. Bruun, G. M. \& Taylor, E. Quantum phases of a two-dimensional dipolar fermi gas. Phys. Rev. Lett. 101, 245301 (2008).

25. Levinsen, J., Cooper, N. R. \& Shlyapnikov, G. V. Topological $p_{x}+i p_{y}$ superfluid phase of fermionic polar molecules. Phys. Rev. A 84, 013603 (2011).

26. Potter, A. C., Berg, E., Wang, D. W., Halperin, B. I. \& Demler, E. Superfluidity and Dimerization in a Multilayered System of Fermionic Polar Molecules. Phys. Rev. Lett. 105, 220406 (2010).

27. Lutchyn, R. M., Rossi, E. \& Das Sarma, S. Spontaneous interlayer superfluidity in bilayer systems of cold polar molecules. Phys. Rev. A 82, 061604 (2010).

28. Samokhin, K. V. \& Mar'Enko, M. S. Nonuniform mixed-parity superfluid state in fermi gases. Phys. Rev. Lett. 97, 197003 (2006)

29. Wu, C. \& Hirsch, J. E. Mixed triplet and singlet pairing in ultracold multicomponent fermion systems with dipolar interactions. Phys. Rev. B 81, 020508 (2010).

30. Shi, T., Zhang, J. N., Sun, C. P. \& Yi, S. Singlet and triplet bes pairs in a gas of twospecies fermionic polar molecules. arXiv: 0910.4051 (2009).

31. Kain, B. \& Ling, H. Y. Singlet and triplet superfluid competition in a mixture of two-component fermi and one-component dipolar bose gases. Phys. Rev. A 83, 061603 (2011).

32. Anderson, P. W. \& Morel, P. Generalized bardeen-cooper-schrieffer states and the proposed low-temperature phase of liquid $\mathrm{He}^{3}$. Phys. Rev. 123, 1911 (1961).

33. Balian, R. \& Werthamer, N. R. Superconductivity with pairs in a relative $p$ wave. Phys. Rev. 131, 1553 (1963).

34. Brinkman, W. F., Serene, J. W. \& Anderson, P. W. Spin-fluctuation stabilization of anisotropic superfluid states. Phys. Rev. A 10, 2386 (1974).

35. Leggett, T. A theoretical description of the new phases of liquid ${ }^{3} \mathrm{He}$. Rev. Mod. Phys. 47, 331 (1975)

36. Volovik, G. E. The Universe in a Helium droplet. Oxford University Press (2009).

37. Ohmi, T. \& Machida, K. Bose-Einstein condensation with internal degrees of freedom in alkali atom gases J. Phys. Soc. Jpn. 67, 1822 (1998).

38. Ho, T. L. Spinor bose condensates in optical traps. Phys. Rev. Lett. 81, 742-745 (1998).

39. Zhou, F. Quantum spin nematic states in bose einstein condensates. Int. J. Mod. Phys. B 17, 2643-2698 (2003)

40. Demler, E. \& Zhou, F. Spinor bosonic atoms in optical lattices: symmetry breaking and fractionalization. Phys. Rev. Lett. 88, 163001 (2002)

41. Lu, M., Burdick, N. Q. \& Lev, B. L. Quantum degenerate dipolar Fermi gas. arXiv: 1202.4444

42. Cheng, M., Sun, K., Galitski, V. \& Das Sarma, S. Stable topological superconductivity in a family of two-dimensional fermion models. Phys. Rev. B 81, 024504 (2010).

\section{Acknowledgement}

C. W. thanks J. E. Hirsch for helpful discussions. Y. L and C. W. are supported by NSF under No. DMR-1105945, and the AFOSR YIP program.

\section{Author contributions}

Both authors participated in the research and in the writing of the manuscript.

\section{Additional information}

Competing financial interests: The authors declare no competing financial interests.

License: This work is licensed under a Creative Commons

Attribution-NonCommercial-ShareAlike 3.0 Unported License. To view a copy of this license, visit http://creativecommons.org/licenses/by-nc-sa/3.0/

How to cite this article: $\mathrm{Li}, \mathrm{Y} . \& \mathrm{Wu}, \mathrm{C}$. The $J$-triplet Cooper pairing with magnetic dipolar interactions. Sci. Rep. 2, 392; DOI:10.1038/srep00392 (2012) 